\title{
6 LITERATURVERZEICHNIS
}

Abraham, K. (2001): Interactions between dogs and wildlife in parks on the Berkeley Marina. Unpublished Report, submitted to Berkely Parks and Recreation. In: Lenth, B., Brennan, M. \& L. Knight, R. (2006): The Effects of dogs on wildlife communities. In Landscape and Urban Planning 78 (2006) 251-262.

Aeroclub, Österreich (2009): www.aeroclub.at (abgerufen am 20.7.2009).

Akademie für Raumforschung und Landesplanung (Hg.) (1982): Grundriß der Raumordnung. Hannover.

Allex, B. (2008): Ziesel am Bisamberg - Umweltbewusstsein der Besucher und ihre Bereitschaft zur Verhaltensänderung zum Schutz einer bedrohten Art, Diplomarbeit, Universität für Bodenkultur. Wien.

Al-Sahili, K. und M. Aboul-Ella (1992): Accessibility of Public Services in Irbid, Jordan. Journal of Urban Planning and Development, 118.

Ammer \& Pröbstl U. (1991): Freizeit und Natur, Verlag Paul Parey, Hamburg, Berlin.

AMS Arbeitsmarktdaten: http://iambweb.ams.or.at/ambweb/AmbwebServlet?trn=start, 8.2.2011.

Amt der Niederösterreichischen Landesregierung (2004): Strategie Niederösterreich. Landesentwicklungskonzept, St. Pölten. URL: http://www.noe.gv.at/bilder/d10/landesentwicklungskonzept.pdf, 18.7.2011.

Amt der Niederösterreichischen Landesregierung (2005): Perspektiven für die Hauptregionen, St. Pölten.

URL: http://www.noe.gv.at/bilder/d11/perspektiven_fuer_die_hauptregionen.pdf, 18.7.2011.

Amt der Niederösterreichischen Landesregierung (2008): Das Landesentwicklungskonzept für Niederösterreich. URL: http://www.noe.gv.at/Land-Zukunft/Landesentwicklung-StrategieNOe/Landesentwicklungskonzept.html, 18.7.2011.

Amt der Niederösterreichischen Landesregierung: Raumordnung und Regionalpolitik Regionale Raumordnungsprogramme:

http://www.raumordnung-noe.at/dynamisch/showcontainer.php?id=45\& (abgerufen am 25.7.2011).

Amt der niederösterreichischen Landesregierung (2007): Stadt Umland Management WienNiederösterreich:

http://www.noe.gv.at/Politik-Verwaltung/Behoerdenwegweiser/RegionalmanagementNiederoesterreich/Regionalmanagement_KontaktSUM.wai.html (abgerufen am: 24.7.2011).

Andersen R, Linnell JDC, Langvatn R (1996): Short term behavioural and physiological re-sponse of moose Alces alces to military disturbance in Norway. Biol Conserv 77: 169-176.

Anderson D.W. \& Keith J.O. (1980): The human influence on seabird nesting success: Conservation implications. Biol Conserv 18: 65-80.

Antos MJ, Ehmke GC, Tzaros CL, Weston MA (2007): Unauthorised human use of an urban coastal wetland sanctuary: Current and future patterns. Landscape and Urban Planning 80: 173-183.

Arbeitsgemeinschaft Rechtsgrundlagen für Fuss- und Wanderwege [ARF] (1982): Fusswege im Siedlungsbereich. Richtlinien für bessere Fussgängeranlagen, Zürich.

ARGE KREK / Raum Mödling (2003): Kleinregionales Entwicklungskonzept Raum Mödling. Kurzbericht. URL: 
http://moedling.riskommunal.net/system/web/zusatzseite.aspx?menuonr=221166367\&detailon $r=221165130$ (abgerufen am:12.10.2011).

ARGE MTB NÖ (2008): http://mbike.at/mbike/noe

Arroyo B, Razin M (2006): Effect of human activities on bearded vulture behaviour and breed-ing success in the French Pyrenees. Biol Conserv 128: 276-284.

Auer M. (1982): Wiesengesellschaften im Wienerwald. Diplomarbeit, Universität für Bodenkultur, Wien.

Austrian Geocache Database, www.aj-gps.at (letzter Zugriff 09.2010).

Banzhaf \& Netzband (2000): Analyses and evaluation of nature space potential in peri- urban spaces using remote sensing data and GIS, - In: International Archives of Photogrammetry and Remote Sensing, Vol. XXXIII, Part B 7/1, Comm. VII, Amsterdam, The Netherlands, 2000, pp.118-125. Banzhaf_Netzband_2000.pdf.

Barja I, Silv n G, Rosellini S, Pi土eiro A, Gonz lez-Gil A, Camacho L, Illera JC (2007): Stress physiological responses to tourist pressure in a wild population of European pine marten. The Journal of Steroid Biochemistry and Molecular Biology 104: 136-142.

Barsch, Heiner, Saupe, Gabriele et al. (1994): Bewertung und Gestaltung der naturnahen Landschaft in Schutzgebieten, Erholungs- und Freizeitgebieten, Teil 1 bis 3 (Anhang). Potsdam (Potsdamer Geographische Forschungen, 8).

Bastian O., \& Schreiber K.F. (1994) Analyse und ökologische Bewertung der Landschaft, Jena/ Stuttgart.

Baydack, R. K. (1986): Sharp-tailed grouse response to lek disturbance in the Carberry Sand Hills of Manitoba. Colorado State University, Fort Collins, Colorado. In: Sime, C.A. (1999): Domestic Dogs in Wildlife Habitats. In: Joslin G., Yousmans H (Hrsg.) (1999): Effects of recreation on Rocky Mountains wildlife: A review for Montana. Committee on Effects of Recreation on Wildlife, Montana Chapter of The Wildlife Society

Bayrisches Staatsministerium des Innern (1996): Wohnumfeld- Gestalt und Nutzung wohnungsnaher Freiräume, Arbeitsblätter für die Bauleitplanung Nr.10, Grünordnung und Landschaftspflege.

Beale CM, Monaghan P (2004): Behavioural responses to human disturbance: a matter of choice? Animal Behaviour 68: 1065-1069.

Beauchamp G (2006): Nonrandom patterns of vigilance in flocks of the greater flamingo, Phoenicopterus ruber ruber. Animal Behaviour 71: 593-598.

Bender LG, Beyer DE, Haufler JB (1999): Effects of short-duration, high-intensity hunting on elk wariness in Michigan. Wildlife Society Bulletin 27: 441-445.

Berg- und Naturwacht, www.bergundnaturwacht.at (letzter Zugriff: 26.01.2011).

Berger, Alexander (2003): Langzeitmonitoring einer Landschaft mittels GIS - Landschafts-wandel \& Erholungswert. Diplomarbeit TU Dresden. $105 \mathrm{~S}$. (nicht eingesehen, siehe WALZ/BERGER 2004).

Berkel, Karl (1984): Konfliktforschung und Konfliktbewältigung. Ein organisations-psychologischer Ansatz, Berlin.

Berkel, Karl (1997): Konflikttraining. Konflikte verstehen, analysieren, bewältigen, 5. neubearbeitete und erweiterte Auflage, Heidelberg (Arbeitshefte für Führungspsychologie, Band 15).

Bezirk Mödling - Die Grünen: Kleinregionales Entwicklungskonzept für den Raum Mödling: http://bezirk.moedling.gruene.at/artikel_grundsaetzlich/kleinregionales_entwicklungskonzept_f uer_den_raum_moedling/ (abgerufen am: 12.10.2011). 
BFN - Bundesamt für Naturschutz (2011): Natursportinfo. Informationssystem des Bundes-amtes für Naturschutz BfN (D) in Zusammenarbeit mit dem Bundesamt für Umwelt BAFU (CH). http://www.bfn.de/natursport/info/ (aufgerufen am 29.11.2011).

Bhat, G., Bergstrom J. (1997): Integration of Geographical Information System Based Spatial Analysis in Recreational Demand Analysis, Faculty Series, University of Georgia.

Billion, F. \& Flückiger,B. (1978): Bedarfsanalyse Naherholung und Kurzzeittourismus. Bremen. In: Schnell, P. (2004): Tagesausflugsverkehr. In Becker C., Hopfinger, H., Steinecke, A. (2004): Geographie der Freizeit und des Tourismus: Bilanz und Ausblick, 2. Auflage, R. Ouldenbourg Verlag.

BKA \& BMLFUW (2008): Standards der Öffentlichkeitsbeteiligung. Empfehlungen für die gute Praxis. Herausgegeben vom Bundeskanzleramt Österreich und dem Lebensministerium. Vom Ministerrat beschlossen am 2. Juli 2008. Wien.

http://www.partizipation.at/fileadmin/media_data/Downloads/Standards_OeB/standards_der_o effentlichkeitsbeteiligung_2008_druck.pdf

BKA \& BMLFUW (2011): Standards der Öffentlichkeitsbeteiligung. Praxisleitfaden. Herausgegeben vom Bundeskanzleramt Österreich und dem Lebensministerium. Wien.

Blumstein DT (2006): Developing an evolutionary ecology of fear: how life history and natural history traits affect disturbance tolerance in birds. Animal Behaviour 71: 389-399.

BMLFUW (2006): Fairplay im Wald.

BMVIT \& ÖFGS (2007): RVS 04.03.12 Wildschutz. Herausgegeben vom Bundesministerium für Verkehr, Infrastruktur und Technologie sowie von der Österreichischen Forschungsgesellschaft Straße - Schiene - Verkehr.

Boldt A. \& Magun B. (2009): Berechnung eines integralen und artspezifischen Konfliktpotentials als Grundlage der Störungsvermeidung, www.faunalpin.ch/files/u2/Poster_Methode_KPot.pdf. (letzter Zugriff: 09.2011).

Boldt A. et al., 2010, Überarbeitung der Wildschutzgebiete im Kanton Bern, Begleitende Aspekte zur Umsetzung der empfohlenen Massnahmen Bericht zuhanden des Jagdinspektorat des Kantons Bern.

Bolduc F, Guillemette M (2003): Human disturbance and nesting success of Common Eiders: interaction between visitors and gulls. Biol Conserv 110: 77-83

Bonertz, J. (1981): Die Planungstauglichkeit von Landschaftsbewertungsverfahren in der Landesund Regionalplanung, Trier (Materialien für Fremdenverkehrsgeographie, Heft 7).

Boroski BB, Mossman AS (1998): Water use patterns of mule deer (Odocoileus hemionus) and the effects of human disturbance. Journal of Arid Environments 38: 561-569.

Boutin, S. (1990): Food supplementation experiments with terrestrial vertebrates: Patterns, problems and the future. Canadian Journal of Zoology, 68(2), 203-220. In: Orams. M. B. (2002) Feeding wildlife as a tourism attraction: a review of issues and impacts. Tourism Management 23 (3):281-293

Bowles, A. E. (1995): Responses of wildlife to noise. Pages 109-156. In: Knight, R. \& K. Gutzwiller (Hrsg.) (2006) Wildlife and recreationists: coexistence through management and research. Island Press. Washington, D.C., USA.

Brandli, U.B., Ulmer, U. (2001): Recreational function. In: Brassel, P., Lischke, H. (eds.), Swiss National Forest inventory: Methods and models of the second assessment. Swiss Federal Research Institute WSL, Birmensdorf.

Brickner, I. (2002): The impact of domestic dogs (Canis familiaris) on wildlife welfare and conservation: a literatur review. With a situation summary from Israel. Unpublished report. Online abrufbar unter: wwww.tau.ac.il/lifesci/zoology/members/yom-tov/inbal/dogs.pdf. 
Bruns, Elke (2007): Bewertungs- und Bilanzierungsmethoden in der Eingriffsregelung: Analyse und Systematisierung von Verfahren und Vorgehensweisen des Bundes und der Länder, Berlin, veröffentlicht unter http://deposit.ddb.de/cgi-bin/dokserv?idn=984579451\&dok _var=d1\&dok_ext=pdf\&filename=984579451.pdf (abgerufen am:5.11.2009).

Bundesamt für Bauwesen und Raumordnung (2005): Raumordnungsbericht 2005, Bonn, S. 209/20.

Bundesamt für Raumentwicklung ARE (2006): Raumstruktur und Mobilität von Personen, Ergebnisse einer Sonderauswertung des Mikrozensus 2000 zum Verkehrsverhalten - Technischer Arbeitsbericht -

Bundesforstgesetz 1996, BGBI. Nr. 793/1996, idF. 2000.

Burda Community Network GmbH: Typologie der Wünsche 2006/2007, online abrufbar unter: http://de.statista.com/statistik/diagramm/studie/30379/umfrage/joggen-laufen-in-derfreizeit/\#info.

Bürg, J., Ottitsch, A. \& Pregernig, M. (1999): Die Wiener und ihre Wälder - Zusammenfassende Analyse sozioökonomischer Erhebungen über die Beziehung der Wiener Stadtbevölkerung zu Wald und Walderholung. Projektendbericht an die Magistratsabteilung 49 (Forstamt und Landwirtschaftsbetrieb) der Stadt Wien.

Burger J (1981): The effect of human activity on birds at a coastal bay. Biol Conserv 21: 231-241.

Canalys (2007): Worldwide mobile navigation device market more than doubles. Online abrufbar unter: http://www.canalys.com/pr/2007/r2007083.pdf.

Cano Alonso LS (2006): An approach to wintering of Black Stork Ciconia nigra in the Iberian Peninsula. Biota 7: 7-13.

Caro TM (1994): Ungulate antipredator behaviour: preliminary and comparative data from African bovids. Behaviour 128: 189-228.

Carr M.H. \& Zwick P (2005): Using GIS suitability analysis to identify potential future land use conflicts in North Central Florida, Journal of Conservation Planning Vol 1 (2005) 58-73.

CENTROPE - region centrope - CENTROPE auf einen Blick: http://www.centrope.com/de/region-centrope/centrope-auf-einen-blick (abgerufen am: 19.6.2011).

Cermak, P. (2008): Perchtoldsdorf - Stadtrandgemeinde mit Funktionsziel der Stadtnaherholung und Auswirkungen des Erholungsverkehrs auf die Gemeinde. Diplomarbeit. Wirtschaftsuniversität Wien.

Cessford, G. (1995): Off-road mountain biking: a profile of participants and their recreation setting and experience preferences, New Zeeland

Cessford, G. (2002): Perception and Reality of Conflict: Walkers and Mountainbikers on the Queen Charlotte Track in New Zealand. Conference Proceedings; Monitoring and Management of Visitor Flows in Recreational and Protected Areas, Universität für Bodenkultur, Wien, 30.Jänner-2.Februar 2002.

Chavez, D.J., Courtright, R. \& Schneider, I. (2004): Over the river and through the woods. Parks \& Recreation, 39, 4, 68-72.

Chavez, D.J., Schneider, I. \& Powell, T. (2004): The social-psychology of a technology driven outdoor trend: Geocaching in the USA. [Proceedings paper]. Hawaii International Conference on Social Sciences; 2004 June 16-19; Honolulu, HI.

Chevallier D, Baillon F, Robin JP, Le Maho Y, Massemin-Challet S (2008): Prey selection of the black stork in the African wintering area. J Zool 276: 276-284. 
Christensen NL (1988): Succession and natural disturbance: Paradigms, problems and preservation of natural ecosystems. In Ecosystem manangement for parks and wilderness., Agee JK, Darryll RJ (eds) pp 62-86. University of Washington Press.: Seattle and London.

Clair CC, Forrest A (2009): Impacts of vehicle traffic on the distribution and behaviour of rut-ting elk, Cervus elaphus. Behaviour 146: 393-413.

Clawson, M. and Knetsch, J.L. (1966): Economics of outdoor recreation. Resources for the future, Inc., Washington D.C. (2nd edition 1969).

Cole D.N. \& Landres P.B. (1995): Indirect effects of recreation on wildlife. In: Knight, R.L., Gutzwiller K.J.(Hrsg) (2006): Wildlife and Recreationists: Coexistence Through Management and Research. Washington, pp183-202.

Cooper J, Frederick WG (2007): Optimal flight initiation distance. Journal of Theoretical Biol-ogy 244: 59-67.

Cooper Jr (2005): When and how do predator starting distances affect flight initiation distanc-es? Can J Zool 83: 1045-1050.

Council of Europe (2000): European Landscape Convention. online unter http://www.coe.int/t/dg4/cultureheritage/heritage/Landscape/default_en.asp, (letzter Zugriff: 27.01.2011).

Czech B (1991): Elk behavior in response to human disturbance at Mount St. Helens National Volcanic Monument. Appl Anim Behav Sci 29: 269-277.

Bundesinstitut für Bau-, Stadt- und Raumforschung (BBSR): Raumordnungsbericht 2005: http://www.bbsr.bund.de/cln_016/nn_23502/BBSR/DE/Veroeffentlichungen/Berichte/2000_20 05/Bd21Raumordnungsbericht2005.html (abgerufen am:15.11.2009).

Datta T, Pal BC (1993): The effect of human interference on the nesting of the openbill stork Anastomus oscitans at the raiganj wildlife sanctuary, India. Biol Conserv 64: 149-154.

DeBoer HY, VanBreukelen L, Hootsmans MJM, VanWieren S.E. (2004): Flight distance in roe deer Capreolus capreolus and fallow deer Dama dama as related to hunting and other factors. Wildl Biol 10: 35-41.

Deutsche Gesellschaft für Freizeit (Hrsg) (o.J): Freizeitlexikon. Ostfildern.

Deutscher Aero Club e.V., Bundesamt für Naturschutz (2003): Luftsport und Naturschutz - Gemeinsam abheben, Braunschweig, 2003

Deutscher Aero Club e.V., Bundesamt für Naturschutz, Deutscher Hängegleiterverband e.V. (Hrsg) (2009): Arbeitsunterlagen Luftfahrt und Naturschutz.

Deutsches Wissenschaftliches Institut für Fremdenverkehr (1995): Tagesreisen der Deutschen. Schriftenreihe des DWIF, Heft 46. München 1995.

DHV (Deutscher Heilbäderverband) (2005): Begriffsbestimmungen - Qualitätsstandards für die Prädikatisierung von Kurorten, Erholungsorten und Heilbrunnen, 12.Auflage.

Die Presse", Print-Ausgabe, 09.09.2008. Online abrufbar unter: http://diepresse.com/home/panorama/oesterreich/412552/index.do.

Donadio E, Buskirk SW (2006): Flight behavior in guanacos and vicunjas in areas with and without poaching in western Argentina. Biol Conserv 127: 139-145.

Drum, M. (1981): Hinterhöfe - Gartenhöfe in gründerzeitlicher Blockbebauung, in: Informationen zur Raumentwicklung 7,8/1981.

Durden, L. A. \& N. Wilson (1990): Ectoparasitic and phoretic arthropods of Virginia opossums (Didelphis virginiana) in central Tennessee. Journal of Parasitology 76(4):581-583. In: Sime, C.A. (1999): Domestic Dogs in Wildlife Habitats. In: Joslin G., Yousmans H (Hrsg) (1999): Ef- 
fects of recreation on Rocky Mountains wildlife: A review for Montana. Committee on Effects of Recreation on Wildlife, Montana Chapter of the Wildlife Society.

DWV - Deutscher Wanderverband (2011): Digitale Wanderwegeverwaltung als Beitrag zum ehrenamtlichen Naturschutz; Kooperationsprojekt Naturverträgliches Geocaching. In: Natur und Landschaft, 2011, Sonderausgabe: 45.

Eason PK, Sherman PT, Rankin, Coleman (2006): Factors Affecting Flight Initiation Distance in American Robins. J. Wildl. Manage. 70: 1796-1800.

Eberle, I (o.J.): Flächenangebote und Freizeitinfrastruktur des stadtnahen ländlichen Raums in der BRD und ihrer Nutzung durch den Naherholungsverkehr.

Edwards V. \& Knight, S. (2006): Understanding the Psychology of Walkers with Dogs: new approaches to better management. University of Portsmouth.

Ekanayake \& Dayawansa, 2003, Land suitability identification for a production forest through GIS techniques. Map India Conference 2003, Indien

Ellenberg U, Mattern T, Seddon PJ, Jorquera GL (2006): Physiological and reproductive consequences of human disturbance in Humboldt penguins: The need for species-specific visitor management. Biol Conserv 133: 95-106.

Engels, Barbara (2008): Räumliche Ansprüche des Sektors „Freizeit und Tourismus“ an Natur und Landschaft. In: Institut für Technikfolgenabschätzung und Systemanalyse (ITAS) (Hsg.) Technikfolgenabschätzung - Theorie und Praxis, Nr. 2, 17. Jahrgang - September 2008, S. 52-59, veröffentlicht unter http://www.itas.fzk.de/tatup/082/enge08a.htm (abgerufen am: 1.11.2009).

Europäische Kommission (2008): Leitfaden zu den Jagdbestimmungen der Richtlinie 79/409/EWG des Rates über die Erhaltung der wild lebenden Vogelarten. Februar 2008.

Evert, Klaus-Jürgen (Hrsg.) (2001): Lexikon Landschafts- und Stadtplanung: mehrsprachiges Wörterbuch über Planung, Gestaltung und Schutz der Umwelt = Dictionary landscape and urban planning, Berlin, S. 188-191.

FAO (1985) Guidelines: Land evaluation for irrigated agriculture, FAO Soils Bull, 55.

Fassmann, H., Görgl, P. und M. Helbich (2009): Atlas der wachsenden Stadtregion. Materialienband zum Modul 1 des Projekts „Strategien zur räumlichen Entwicklung der Ostregion (SRO)“, Wien. URL:

http://www.pgo.wien.at/download/PGO_Atlas\%20der\%20wachsenden\%20Stadtregion.pdf (abgerufen am: 20.10.2010).

Faunalpin, www.faunalpin.ch (letzter Zugriff: 08.2011).

Foin, T.C., Garton, E.O., Bowen, C.W., Everingham, J.M., Schultz,R.O. \& Holton, B. (1977): Quantitative studies of visitor impacts on environments of Yosemite National Park, California, and their implications for park management policy. J. Environ. Manage. In: Piper S.D. \& Catterall, C.P. (2006): Impacts of picnic areas on bird assemblages and nest predation activity within Australian eucalypt forests. In Landscape and Urban Planning 78 (2006) 251-262.

Forum Umweltbildung: LandschaftsBildungsLandschaft - Glossar: http://www.umweltbildung.at/LBL/allgemein/index_glossar.htm (abgerufen am: 5.11.2009).

Frank, G. \& Berg, M. (2001): Verbreitung und Schutz des Schwarzstorchs (Ciconia nigra) im Wienerwald. Ein Gemeinschaftsprojekt von BirdLife Österreich, der Österreichischen Bundesforste AG und des Österreichischen Naturschutzbundes. BirdLife Österreich - Gesellschaft für Vogelkunde. Wien.

Froitzheim T., Spittler R. (1997): Leitbilder eines natur- und landschaftsverträglichen Mountainbikings, Bielefeld.

Gaudy, D. (2003): Mit dem Hund zu Gast in der Natur. Schweizer Hunde Magazin 4/03. 
Geist C, Liao J, Libby S., Blumstein DT (2005) Does intruder group size and orientatiion affect flight initiation distance in birds? Animal Biodiversity and Conservation 28: 69-73.

Geocaching - Das österreichische Portal (2009): www.geocache.at (abgerufen am 30.04.2009).

Geocaching - The Official Global GPS Cache Hunt Site (2009): www.geocaching.com (abgerufen am 21.09.2009).

Geocaching in Deutschland (2009): www.geocaching.de (abgerufen am 30.04.2009).

Geological Society of America (2009): http://www.geosociety.org/Earthcache/

George SL, Crooks KR (2006): Recreation and large mammal activity in an urban nature reserve. Biol Conserv 133: 107-117.

George SL, Crooks KR (2006): Recreation and large mammal activity in an urban nature reserve. Biol Conserv 133: 107-117.

Georgii, B. \& Hofer, D. (1997): Ballonsport, Tiere und Vegetation. Materialien 123. Bayrisches Staatsministerium für Landesentwicklung und Umweltfragen, München. In: Komenda-Zehnder, S. \& Brudererer, B. (2002): Einfluss des Flugverkehrs auf die Avifauna. Literaturstudie. Schriftenreihe Umwelt Nr. 344. Bundesamt für Umwelt, Wald und Landschaft, Bern.

Georgii, B.\& Hofer D., Zeitler A. (1994): Hängegleiten, Gleitsegeln und Wildtiere - Eine Umfrage unter Piloten, Berufsjägern, Naturschützern und Bergsteigern. Verh. Ges. Ökologie 23.263267.

Gimblet H.R. (2002): Integrating geographic information systems and agent-based modeling techniques for simulating social and ecological processes, Oxford University Press.

Glasl, F. (1999): Konfliktmanagement: Ein Handbuch für Führungskräfte, Beraterinnen und Berater, Bern/Stuttgart.

Görgl, P., Helbich, M., Fassmann, H. und V. Gazdek (2010): Wanderungsströme der Bevölkerung in der Stadtregion Wien. In: Schrenk, Manfred, Popovich, Vasily V. und Peter Zeile (Hrsg.): REAL CORP 2010 Proceedings/Tagungsband, Vienna, 18. URL: http://www.corp.at (abgerufen am: 14.11.2010).

Gotanda K, Turgeon K, Kramer D (2009) Body size and reserve protection affect flight initia-tion distance in parrotfishes. Behavioral Ecology and Sociobiology.

Grabaum, R., Meyer, B.C., Friedrich, K.E., Wolf, T., Meyer, T. und J. Gehrung (2005): Interaktives Nutzerhandbuch für das Verfahren MULBO - Textdokumente - Bewertungshandbuch Sozio-ökonomische Bewertungsverfahren (OLANIS Expertensysteme $\mathrm{GmbH}$ 2005), veröffentlicht unter http://www.mulbo.de/download/bewert_soziooeko.pdf (abgerufen am: 8.8.2009).

Green, R., Giese, M. (2004): Negative effects of wildlife tourism on wildlife. In: Higginbottom, K. (Ed.): Wildlife Tourism: Impacts, Management and Planning. CRC for Sustainable Tourism and Common Ground Publishing, Altona, Vic., pp. 81-97. Zitiert in: Piper SD., Catterall, CP (2006): Impacts of picnic areas on bird assemblages and nest predation activity within Australian eucalypt forests. In Landscape and Urban Planning 78 (2006) 251-262.

Greif, F., Pfusterschmid, Sophie und Klaus Wagner (2002): Beiträge zur Landwirtschaftlichen Raumplanung, Schriftenreihe der Bundesanstalt für Agrarwirtschaft Nr. 93, Wien, veröffentlicht unter https://www.dafne.at/prod/dafne_plus_common/attachment_download/b427fd19cfa378c49c5b 4f9e3b6961bb/Interreg\%20IIC_Struktur_Bodennutzung.pdf (abgerufen am: 18.11.2009).

Greif, Franz, Pfusterschmid, Sophie und Klaus Wagner (2003): Die Planung ländlicher Kulturlandschaften - eine Zukunftsaufgabe. In Ländlicher Raum 3 / 2003, veröffentlicht unter http//:www.laendlicher-raum.at/filemanager/download/33443/ (abgerufen am: 1.11.2009).

Gründel, M. (2007): Geocaching. Basiswissen für draußen, Band 203. 
Gunduz A, Turedi S, Nuhoglu I, Kalkan A, Turkmen S (2007) Wild Boar Attacks. Wilderness and Environmental Medicine 18: 117-119

Hahn, P. (1992): Der Freizeit-Knigge. Ratgeber für umweltfreundliche Freizeitgestaltung. Düsseldorf, Köln: Bund Verlag.

Hammerich, Müller \& Schaffrath (1995): Natur: Zwiาschen Schutz und Nutzung, Sankt Augustin (Naturschutz und Freizeitgesellschaft, Band 1).

Hammit D.E. \& Cole D.N.(1998): Wildland Recreation. Ecology and Management, Canada.

Hampl et al. (2005): Food provisioning and nestling diet of the Black Stork in the Czech Republic. Waterbirds 28: 35-40.

Hanika, Alexander (2010): Kleinräumige Bevölkerungsprognose für Österreich 2010-2030 mit Ausblick bis 2050 („ÖROK-Prognosen“) Teil 1: Endbericht zur Bevölkerungsprognose, Wien. URL:

http://www.statistik.at/web_de/statistiken/bevoelkerung/demographische_prognosen/bevoelker ungsprognosen/index.htmi \#index2 (abgerufen am: 22.11.2010).

Harfst, Wolfgang (1980): Zur Gültigkeit von Erholungsbewertungsmethoden. Kritische Analyse derzeitiger Verfahrensansätze als Instrumente der Landschaftsplanung, Hannover.

Harveson PM, Lopez RR, Collier BA, Silvy NJ (2007): Impacts of urbanization on Florida Key deer behavior and population dynamics. Biol Conserv 134: 321-331.

Hay Smith, L., \& J.D. Hunt. (1995): Nature tourism: impacts and management. In Knight R. L. \&. Gutzwiller K.J (Hrsg) (1995). Wildlife and Recreationists: Coexistence Through Management and Research. Island Press, Washington, DC.

Heimtierdatenbank NÖ, http://heimtierdatenbank.ehealth.gv.at, letzter Zugriff: 08.2010.

Heinze W. G. \& Kill H.H (1997): Freizeit und Mobilität - Neue Lösungen im Freizeitverkehr, ARL Hannover. In: Wolf A. \& Appel-Kummer E. (2009): Naherholung in Stadt und Land, Norderstedt, Books on Demand.

Hennig, R. (2001): Schwarzwild: Biologie, Verhalten, Hege und Jagd. BLV Verlagsgesellschaft $\mathrm{mbH}$ : München Wien Zürich.

Henry, J.D. (1977): Use of urine marking in scavenging behavior of red foxes, Vulpes vulpes. Behaviour 61:82-106. In Lenth, B., Brennan, M. \& Knight, R.L. (2006): The Effects of dogs on wildlife communities.

Hernandez L, Laundre JW (2005): Foraging in the 'landscape of fear' and it's implications for habitat use and diet quality of elk Cervus elaphus and bison Bison bison. Wildl Biol 11: 245-220.

Herzog, S. (2000): Genetische Strukturen des Rotwildes (Cervus elaphus) in Westdeutschland und deren Bedeutung im Rahmen innovativer Managementkonzepte. Zeitschrift für Jagdwissenschaft 46: 188-192.

Hespeler, B. (2004): Scharzwild Heute. Lebensweise - Schadensbegrenzung - Ansprechen Jagdarten - Wildbretverwertung. BLV Verlagsgesellschaft München Wien Zürich: München.

Hinterberger, F. \& Schnepf, D. (2002): Arbeit und Ökologie - Vorstudie für eine ökosoziale Reformstrategie zur Zukunft von Arbeit und Ökologie in Österreich, BMLFUW, Wien. In: Wolf A. \& Appel-Kummer E. (2009): Naherholung in Stadt und Land, Norderstedt, Books on Demand.

Hinterstoisser, H., Jerabek, M. \& Stadler, S. (2006): Besucherlenkung in Schutzgebieten: Lösungsansätze für ein Miteinander unterschiedlicher Natur-Nutzergruppen. Naturschutzbeiträge $32 / 06$.

Hodgetts BV, Waas JR, Matthews LR (1998): The effects of visual and auditory disturbance on the behaviour of red deer (Cervus elaphus) at pasture with and without shelter. Appl Anim Behav Sci 55: 337-351. 
Hoffmann IE, Milesi E, Pieta K, Dittami JP (2003): Anthropogenic effects on the population ecology of European ground squirrels (Spermophilus citellus) at the periphery of their geo-graphic range. Mammalian Biology - Zeitschrift fur Saugetierkunde 68: 205-213.

Hoffmann, I. E. (2002): Grundlagenerhebung zum Artenschutzprojekt Ziesel, im Auftrag des Magistrats der Stadt Wien, MA 22 - Naturschutzreferat.

Hoisl et al. (2000): Naturbezogene Erholung und Landschaftsbild, Kuratorium für Technik und Bauwesen in der Landwirtschaft E.V., Darmstadt.

Holzner, W.; Bogner, D.; Geburek, T.; Tiefenbach, M. \& Zech, S. (2006): MOBI-e - Entwicklung eines Konzeptes für ein Biodiversitäts-Monitoring in Österreich. Studie im Auftrag des Lebensministerium. Wien.

http://www.planungsverband.de/media/custom/1169_651_1.PDF?1144980611 (abgerufen am: 5.11.2009).

http://www.planungsverband.de/media/custom/1169_652_1.PDF?1144980611 (abgerufen am: 12.4.2010).

http://www.planungsverband.de/media/custom/1169_653_1.PDF?1144980611 (abgerufen am: 5.11.2009).

http://www.raumordnung-noe.at/dynamisch/showcontainer.php?id=45\& (abgerufen am: 25.7.2011).

http://www.zgb.de/barrierefrei/misc/downloads/RROP2008_BeschreibendeDarstellung.pdf (abgerufen am: 2.11.2009).

http://www2.ioer.de/recherche/pdf/2004_walz_berger_agit.pdf (abgerufen am: 1.11.2009).

Hulova F, Sedlacek F (2008): Population genetic structure of the European ground squirrel in the Czech Republic. Conservation Genetics 9: 615-625.

Ingold, P. \& Sutter E. (Hrsg.)(1986): Verhalten und Verhaltensökologie. Ornithologische Beobachtungen. In: Scherzinger, W. (2003): Artenschutzprojekt Auerhuhn im Nationalpark Bayrischer Wald von 1995-2000.

Ingold, P. (2005): Freizeitaktivitäten im Lebensraum der Alpentiere. Bern: Haupt Verlag.

Janssen G, Hormann M, Rohde C (2004): Der Schwarzstorch. Westarp Wissenschaften Verlagsgesellschaft $\mathrm{mbH}$ : Hohenwartsleben.

Jayakody S, Sibbald AM, Gordon IJ, Lambin X (2008): Red deer Cervus elephus vigilance behaviour differs with habitat and type of human disturbance. Wildl Biol 14: 81-91.

Jedrzejewski W, Spaedtke H, Kamler JF, Jedrzejewska B, Stenkewitz U (2006). Group Size Dynamics of Red Deer in Bialowieza Primeval Forest, Poland. J Wildl Manage 70: 1054-1059.

Jiang G, Zhang M, Ma J (2008). Habitat use and separation between red deer Cervus elaphus xanthopygus and roe deer Capreolus pygargus bedfordi in relation to human disturbance in the Wandashan Mountains, northeastern China. Wildl Biol 14: 92-100.

Jones, D., Nealson T. ( 2005): Impacts of bird watching on communities and species: Long-term and short-term responses in rainforest and eucalypt habitats. CRC Sustainable Tourism Technical Reports, Vol. 2005, pp. 1-17.

Kaspar, C. (1996): Tourismuslehre im Grundriss.

Kaufmann, V. (2000): Mobilité quotidienne et dynamiques urbaines. La question du report modal. Lausanne : Presses polytechniques et universitaires romandes.

Keller, V. (1991): Effects of human disturbance on eider ducklings Somateria mollissima in an estuarine habitat in Scotland. Biological Conservation 58:213-228. In: Sime, C.A. (1999): Domestic Dogs in Wildlife Habitats. In: Joslin G., Yousmans H (Hrsg) (1999): Effects of recreation 
on Rocky Mountains wildlife: A review for Montana. Committee on Effects of Recreation on Wildlife, Montana Chapter of the Wildlife Society.

Kiemstedt H. (1967): Zur Bewertung der Landschaft für die Erholung, Beiträge zur Landschaftspflege, Sonderheft 1, Stuttgart.

Kiemstedt, H., Bechmann, A:, Heitmann, G:, Hoerschelmann, O., Hultsch, E:, Muhs, C:, Meyer, E. (1975): Landschaftsbewertung für Erholung im Sauerland. Teil I: Textband, Dortmund (Schriftenreihe Landes- und Stadtentwicklungsforschung des Landes Nordrhein-Westfalen. Band1.008/l).

Kirchmeir et al. (2004): LIFE-Projekt Schütt-Dobratsch - Endbericht: Maßnahme A.2 Informationssystem für den Managementplan. Studie im Auftrag von: ARGE Naturschutz, Bearbeitung: E.C.O. Institut für Ökologie, Klagenfurt.

Kliskey (2000): Recreation terrain suitability mapping: A spatially explicit methodology for determing, Landscape and Urban Planning, Volume 52, Issue 1, 5 November 2000, Pages 33-43.

Knight, R. \& K. Gutzwiller (Hrsg.) (2006). Wildlife and recreationists: coexistence through management and research. Island Press. Washington, D.C., USA.

Knoflacher, H. (2002). Why is the Traffic Safety in Different Cities so different? Technische Universität Wien. In: Wolf A. \& Appel-Kummer E. (2009): Naherholung in Stadt und Land, Norderstedt, Books on Demand.

Köchli, Daniel (2005a): Multifunktionale nachhaltige Waldnutzung in der Region Greifensee. Darstellung und Vergleich von Waldentwicklungs-Szenarien im Schweizer Mittelland. [Diss. ETH 15975] Birmensdorf, Eidg. Forschungsanstalt für Wald, Schnee und Landschaft WSL. 157 S. [+ Anhang auf CD]

Köchli, Daniel (2005b): Waldbewirtschaftung im Mittelland. Die heutige Waldbewirtschaftung leistet vergleichsweise viel!. In: Wald und Holz - 9(2005), S. 49 ff., veröffentlicht unter http://www.waldwissen.net/themen/umwelt_landschaft/landschafts_raumentwicklung/wsl_greif ensee.pdf (abgerufen am: 30.11.2009).

Komenda-Zehnder, S. \& Brudererer, B. (2002): Einfluss des Flugverkehrs auf die Avifauna. Literaturstudie. Schriftenreihe Umwelt Nr. 344. Bundesamt für Umwelt, Wald und Landschaft, Bern ev.

Kulinat, K. \& Steinecke, A. (1984): Geographie des Freizeitverkehrs und Fremdenverkehr. Wissenschaftliche Buchgesellschaft. In: Becker C., Hopfinger, H., Steinecke, A. (2004): Geographie der Freizeit und des Tourismus: Bilanz und Ausblick, 2. Auflage, R. Ouldenbourg Verlag.

Kulinat, K. (1980): Die Bestimmungsfaktoren großstädtischer Naherholungsräume in der Bundesrepublik Deutschland. Modellansätze und Planungskonsequenzen. In: In Becker C., Hopfinger, H., Steinecke, A. (2004): Geographie der Freizeit und des Tourismus: Bilanz und Ausblick, 2. Auflage, R. Ouldenbourg Verlag.

Kurdoğlu,O. (2002): Kaçkar Dağları Milli Parkı ve yakın çevresinin doğal kaynak yönetimi açısından incelenmesi, PhD Thesis, zitiert in GÖZDE KAYA Zhera, 2006, Developing a GIS based methodology for decision making for multiobjective recreational areas, case study: Eastern Black Sea Region.

Land Niederösterreich (Hrsg.) (2007): Respektiere deine Grenzen. Folder.

Landeshauptstadt Dresden, Umweltamt (Hrsg.) (5/2007): Umweltatlas Dresden -2.10 Bewer-tung der Erholungseignung - Karte und Erläuterungen, veröffentlicht unter http://www.dresden.de/de/08/03/01/04/c_0330.php (abgerufen am: 2.11.2009).

Lang, A. (1988): Lebensraum Wohnumfeld, in: Selbsthilfe und Demokratie im Wohnumfeld, Nr. 23, München 1988. 
Lang, H. (1998): Mountainbiking zwischen Umweltverträglichkeit und gesellschaftlicher Akzeptanz. Eine sozialwissenschaftliche Studie über die Problemsichten im Konfliktbereich „Mountainbike, Tourismus und Umwelt“. Diplomarbeit an der ETH Zürich

Langbein J, Putman RJ (1992): Behavioural Responses of Park Red and Fallow Deer to Disturbance and Effects on Population Performance. Animal Welfare 1: 19-38.

Lenth, B., Brennan, M. \& L. Knight, R. (2006): The Effects of dogs on wildlife communities.

Leinenzwang auf der Perchtoldsdorfer Heide: Plan:

http://www.perchtoldsdorf.at/service/pdfs/perchtoldsdorfer-heide.jpg (abgerufen am: 14.12.2011).

Leser, Helmut (Hrsg) (2005): Diercke Wörterbuch Allgemeine Geografie. Braunschweig.

Longley et al. (2001): Geographical Information Systems and Science. London: Wiley

Lorch, J. (1995): Trendsportarten in den Alpen - Konflikte, rechtliche Reglementierungen, Lösungen. CIPRA Kleine Schriften 12/95. Vaduz.

Lorenz, K. \& Leyhausen P. (1968): Antrieb tierischen und menschlichen Verhaltens. Piper \& Co., München.

LRNI_2009061.pdf (abgerufen am: 25.7.2011.

LRNI_2010037.pdf (abgerufen am: 25.7.2011.

LRNI_2010045.pdf (abgerufen am: 25.7.2011).

Luftfahrtgesetz, BGBI. Nr. 253/1957 i.d.F. BGBI. I Nr. 83/2008.

MacArthur, R.A., Geist V. \& Johnston R.H. (1982): Cardiac and behavioral responses of mountain sheep to human disturbance. Journal of Wildlife Management 46:351-358. In: Lenth, B., Brennan, M. \& Knight, R. L. (2006): The Effects of dogs on wildlife communities.

MacArthur, R.A., R.H. Johnston and V. Geist (1979): Factors influencing heart-rate in freeranging bighorn sheep-physiological approach to the study of wildlife harassment. Canadian Journal of Zoology 57:1020-2021. In: Lenth, B., Brennan, M. \& L. Knight, R. (2006): The Effects of dogs on wildlife communities.

Magistratsabteilung 6 Wien, www.wien.gv.at (letzter Zugriff 2010).

Maier, J. \& Ruppert, K. (1970): Die Leisungskraft einer Fremdenverkehrsgemeinde, Modellanalyse des Marktes Hindelang /Allgäu, Bd. 3 d. WGI-Berichte zur Regionalforschung. München. In: Wolf A. \& Appel-Kummer E. (2009): Naherholung in Stadt und Land, Norderstedt, Books on Demand.

Mainini, B. Neuhaus P. \& P. Ingold (1993): Behavior of marmots (Marmota marmota) under the influence of different hiking activities. Biological Conservation 64:161-164 In: Lenth, B., Brennan, M. \& Knight, R. L. (2006): The Effects of dogs on wildlife communities.

Manor R, Saltz D (2005): Effects of human disturbance on use of space and flight distance of mountain gazelles. J Wildl Manage 69: 1683-1690.

Marks et al. (1992): zitiert in Bastian O., \& Schreiber K.F., 1994, Analyse und ökologische Bewertung der Landschaft, Jena/ Stuttgart.

Maschke, Joachim (2005): Tagesreisen der Deutschen, Schriftenreihe des deutschen wirtschaftwissenschaftlichen Instituts für Fremdenverkehr an der Universität München, München.

Mata C, Hervas I, Herranz J, Suarez F, Malo JE (2008): Are motorway wildlife passages worth building? Vertebrate use of road-crossing structures on a Spanish motorway. J Environ Manage 88: 407-415.

Meyer, B. (1997): Formen der Konfliktregelung: Eine Einführung mit Quellen, Opladen (Friedensund Konfliktforschung, Band 3). 
Mieg, H. A. und M. Näf (2006): Experteninterviews in den Umwelt- und Planungswissenschaften. Eine Einführung und Anleitung, Lengerich.

Miller SG, Knight RL, Miller CK (2001) Wildlife Responses to Pedestrians and Dogs. Wildlife Society Bulletin 29: 124-132

Miller, S.G., Knight R.L. \& Miller C.K. (2001): Wildlife response to pedestrians and dogs. Wildlife Society Bulletin 29:124-132. In: Lenth, B., Brennan, M. \& Knight, R. L. (2006): The Effects of dogs on wildlife communities.

Mönnecke, Margit und Karin Wasem (2005): Anleitung zur Berücksichtigung der Naherholung in der kommunalen Planung, Rapperswil, veröffentlicht unter http://www.arpdaten.bl.ch/arpdaten/publikationen/anleitung-naherholung_2006.pdf, 5.11.2009.

Musil, R. \& P. Pindur (2008a): Nachhaltige Suburbanisierung? Entwicklungstrends und Steuerungsmechanismen der Siedlungstätigkeit im Biosphärenpark Wienerwald. Endbericht zum MAB-Forschungsprojekt „Nachhaltige Suburbanisierung?", Wien/Salzburg. URL: http://www.oeaw.ac.at/isr/Personen/publikationen/Projekt_Stille\%20Suburbanisierung.pdf (abgerufen am: 20.10.2010).

Musil, R. \& P. Pindur (2008b): Nachhaltig trotz suburbanen Nutzungsdrucks? Siedlungsentwicklung im Biosphärenpark Wienerwald. In: Mitteilungen der Österreichischen Geographischen Gesellschaft, Wien, S. 99-122. URL: http://arcims.isr.oeaw.ac.at/website/ oegg/publikationen/Bd150_MOEGG/S99_100_Musil.pdf (abgerufen am: 9.1.2011).

NABU (2009):

http://www.nabu.de/tiereundpflanzen/saeugetiere/fledermaeuse/news/06864.html (abgerufen am 30.04.2009).

Nationalparkforstamt Eifel (2005): Konzeption für große störungsarme Räume im Nationalpark Eifel. Abgrenzungsvorschläge für Ruheräume auf Basis vorliegender Untersuchungen (online verfügbar unter:

http://www.nationalparkefel.de/data/inhalt/Stoerungsarme_Raeume_gesamt_2005_2_113394 3453_1136454510.pdf).

Natursportinfo (2009): www.natursportinfo.de.

Niethammer J, Krapp F (1978) Handbuch der Säugetiere Mitteleuropas. Band 1 Nagetiere I. Akademische Verlagsgesellschaft Wiesbaden.

NÖ Biosphärenpark Wienerwald Gesetz: Niederösterreichisches Biosphärenpark Wienerwald Gesetz. LGBI. 5760-0 idF. 2006.

NÖ Jagdgesetz 1974: Niederösterreichisches Jagdgesetz 1974. LGBI. 6500-27 idF. 2011.

NÖ NSG 2000: Niederösterreichisches Naturschutzgesetz 2000. LGBI. 5500-8 idF. 2010.

NÖ Polizeistrafgesetz BGBI. Nr. 135/75, idF. 61/2002.

O’Hara, K. (2008): Understanding Geocaching Practices and Motivations.Proceedings of CHIart.science.balance, Florenz.

ÖIR (2010): Fortschrittsbericht zum STEP'05 im Rahmen der Konferenz „stadtentwicklung geplant\&realisiert" präsentiert. In: ÖIR e-letter Ausgabe 4/2010. URL: http://www.oir.at/files/eletter/4_2010/e_letter_4_2010_step.pdf (abgerufen am: 18.7.2011).

Ökologische bzw. ökologisch orientierte Planung - Lehrmaterial für die Lehrveranstaltung Environmental Planning II an der ETH Zürich, veröffentlicht unter http://www.irl.ethz.ch/plus/education/MSc_level/103-0357-00L/0925_Theorie_Oekologische_ Planung.pdf (abgerufen am: 5.11.2009).

Olliff, T., Legg K. \& Kaeding B. (Hrsg.) (1999): Effects of winter recreation on wildlife of the Greater Yellowstone Area: a literature review and assessment. Report to the Greater Yellowstone Coordinating Committee. Yellowstone National Park, Wyoming. 
Opaschowski H.W. (1997): Einführung in die Freizeitwissenschaft, Opladen.

Opaschowski H.W. (1999): Umwelt.Freizeit.Mobilität. - Konflikte und Konzepte, Opladen.

Orams. M. B. (2002): Feeding wildlife as a tourism attraction: a review of issues and impacts. Tourism Management 23 (3):281-293.

Österreichischer Aero Club e.V. (2009): Statistik 2008; Mitglieder- und Vereinsverwaltung (online verfügbar unter: www.aeroclub.at).

Pearce-Higgins JW, Finney SK, Yalden DW, Langston RHW (2007): Testing the effects of recreational disturbance on two upland breeding waders. Ibis 149: 45-55.

Perchtoldsdorfer Heide: http://www.perchtoldsdorfer-heide.at (abgerufen am: 12.12.2011).

Peters KA, Otis DL (2007): Shorebird roost-site selection at two temporal scales: is human disturbance a factor? Journal of Applied Ecology 44: 196-209.

Pfefferkorn, W; Leitgeb-Zach, M; Heckl, F. \& Gottsberger, T. (2006): Vielfalt statt Zwiespalt. Begleitfaden zum Mitgestalten von Lebensräumen - ein Beitrag zur Umsetzung der Biodiversitäts-konvention. Logos, Berlin.

PGO Planungsgemeinschaft Ost (Hrsg.) (kein Jahr): Jahresbericht 2008, kein Ort.

Phillips GE, Alldredge AW (2000): Reproductive Success of Elk Following Disturbance by Humans during Calving Season. J Wildl Manage 64: 521-530.

Pinkspiral Ballonteam (2009): www.ballonfahrten.at

Piper SD. \& Catterall, CP (2006): Impacts of picnic areas on bird assemblages and nest predation.

PÖTKE, Peter-Michael (1979): Der Freizeitwert einer Landschaft. Quantitative Methode zur Bewertung einer Landschaft für Freizeit und Erholung, Trier (Materialien für Fremdenverkehrsgeographie, Heft 2).

Prahl, H.W. (2002): Soziologie der Freizeit, Leske und Budrich, Opladen.

Pröbstl, U.; Prutsch, A. (2009): Natura 2000 Sport und Tourismus. Ein Leitfaden zur Anwendung der Fauna-Flora-Habitat-Richtlinie und der Vogelschutz-Richtlinie. 78; LV Druck GmbH \&Co.KG, Münster-Hiltrup.

Puchinger, K., Rosenberger, M. und G. Schinko (kein Jahr): Gemeinsame Regionalentwicklungsstrategie für die Region Wien - Bratislava - Györ. JORDES+. Kurzfassung. kein Ort. URL: http://www.pgo.wien.at/jordes_hp/Jordes/05_Projektdokumente_files/Dokumente/Projektdoku mente/kurzfassung_jordes_021010.pdf (abgerufen am: 19.6.2011).

Queensland Government (1998): The Southeast Queensland Outdoor Recreation Study, Queensland Government Brisbane. In: Piper SD. \& Catterall, CP (2006): Impacts of picnic areas on bird assemblages and nest predation activity within Australian eucalypt forests. In: Landscape and Urban Planning 78 (2006) 251-262.

Ranftl, H. (1988): Auswirkungen des Luftsports auf die Vogelwelt und die sich daraus ergebenen Forderungen. In: Berichte der ANL 12/88.

Regional Consulting ZT GmbH (2005): JORDES+ Infoletter3. URL: http://www.pgo.wien.at/pgo_d.html (abgerufen am: 19.6.2011).

Regionalverband NÖ Mitte - Kleinregionen: http://www.netteam.at/opmodule/user/noemitte/?kat=17\&mkat=17\&op=3 (abgerufen am: 25.7.2011).

Reimoser, F.; Lexer, W.; Brandenburg, C.; Zink, R.; Heckl, F.; Bartel, A.; Ferner, B. \& Muhar, A. (2008): Integriertes nachhaltiges Wildtiermanagement im Biosphärenpark Wienerwald. Endbericht des Projekts ISWI-MAB mit Anhängen. Wien, 2008.

http://epub.oeaw.ac.at/6626-9inhalt. 
Reimoser, S. (2012): Influence of Anthropogenic Disturbances on Activity, Behavior and Heart Rate of Roe Deer (Capreolus capreolus) and Red Deer (Cervus elaphus), in Context of their Daily and Yearly Patterns. In: Cahler, A.A, Marsten, J.P (Eds): Deer - Habitat, Behavior and Conservation. Hauptgage. Nova Publishers.

Rowan, A.N. \& Beck, A.M. (1994): The health benefits of human-animal interaction. Anthrozoos, 7(2), 85-8 In: Orams. M. B. (2002): Feeding wildlife as a tourism attraction: a review of issues and impacts. Tourism Management 23 (3): 281-293.

Runningcheckpoint (2009): www.runningcheckpint.at.

RUPPERT, Klaus (1971): Zur Beurteilung der Erholungsfunktion siedlungsnaher Wälder, Frankfurt am Main.

Ruppert,K., Maier, J. (1969): Naherholungsraum und Naherholungsverkehr. Ein sozial und wirtschaftsgeografischer Literaturbericht zum Thema Wochenendtourismus.Starnberg. In: Schnell, P. (2004): Tagesausflugsverkehr. In Beckerc C., Hopfinger, H., Steinecke, A. (2004): Geographie der Freizeit und des Tourismus: Bilanz und Ausblick, 2. Auflage, R. Ouldenbourg Verlag.

Rykiel EJ (1985): Towards a definition of ecological disturbance. Australian Journal of Ecolo-gy 10: 361-365.

Schemel, H.J \& Erbguth, W. (2000): Handbuch Sport und Umwelt.

Schleidt, WM (1961): Reaktionen von Truthühnern auf fliegende Raubvögel und Versuche zur Analyse ihrer AAM's. Z. Tierpsychologie 18: 534-560.

Schnell, P. (2004): Tagesausflugsverkehr. In Beckerc C., Hopfinger, H., Steinecke, A. (2004): Geographie der Freizeit und des Tourismus: Bilanz und Ausblick, 2. Auflage, R. Ouldenbourg Verlag.

Schuster, K.; Brendle, U., Erdmann, K.-H. (2005): Wahrnehmung und Bewusstsein im Naturschutz. In: G. Michelsen \& J. Godemann (Hrsg): Handbuch Nachhaltigkeitskommunikation. Grundlagen und Praxis. S. 405-415. München: ökom.

Sekercioglu, C.H. (2002): Impacts of birdwatching on human and avian communities. In Environmental Conservation 29 (3): 282-289.

Sime, C.A. (1999): Domestic Dogs in Wildlife Habitats. In: Joslin G., Yousmans H (Hrsg.) (1999): Effects of recreation on Rocky Mountains wildlife: A review for Montana. Committee on Effects of Recreation on Wildlife, Montana Chapter of the Wildlife Society.

Speidel, W., Suter C.L. (2006): Forum 3: Mountainbike in geregeltem Rahmen. In: Bundesamt für Naturschutz (2006): Natursport und Kommunikation, Tagungsband zum Internationalen Fachseminar „Erlebnis-Konsumgut Natur: verehrt-verzehrt“, 10-11.11.2005, Basel.

Speight, M.C.D. (1973): Outdoor recreation and its ecological effects: A bibliography and review. Discussion paper in conservation 4. London : University College. In: Hammit D.E. \& Cole D.N. (1998): Wildland Recreation. Ecology and Management, Canada.

Stadt Wien - Der Wiener Grüngürtel: http://www.wien.gv.at/umwelt/natuerlich/100jahre.html (aufgerufen am: 6.6.2011).

Stadt Wien - Stadtentwicklung: http://www.wien.gv.at/stadtentwicklung/ (aufgerufen am: 6.6.2011).

Stadt Wien (Hrsg.) (2007): Der Wiener Hundeführerschein. Mit Sicherheit ein Gewinn. Folder.

Stadtentwicklung Wien, Magistratsabteilung 18 Stadtentwicklung und Stadtplanung (Hrsg.) (2005): STEP 05 Stadtentwicklung Wien 2005. URL:

http://www.wien.gv.at/stadtentwicklung/strategien/step/step05/download/pdf/step-gesamt.pdf (aufgerufen am: 8.6.2011). 
Stankowich T (2008) Ungulate flight responses to human disturbance: A review and metaanalysis. Biol Conserv 141: 2159-2173.

Statistik Austria: http://www.statistik.at (letzter Zugriff 2010).

Statistik Austria - Ein Blick auf die Gemeinde: Niederösterreich: http://www.statistik.at/blickgem/gemList.do?bdl=3 (aufgerufen am: 1.10.2011).

Steiner, F., L. Mc Sherry, and J. Cohen (2000): Land suitability analysis for the Upper Gila River watershed. In: Landscape and Urban Planning 50: 199-455.

Sterl, P., Brandenburg C., Arnberger A. (2008): Visitor awareness and assessment of recreational disturbance of wildlife in the Donau-Auen National Park, Journal of Nature Conservation 16.

Stettler, J. (1997): Sport und Verkehr. Sportmotiviertes Verkehrsverhalten der Schweizer Bevölkerung (Berner Studien zu Freizeit und Tourismus, Bd. 36), 1997, Bern.

Stroh, K. \& Weichwald, S. (2006): Cleverer Umweltschutz - unterwegs in Urlaub und Freizeit. Bayerisches Landesamt für Umwelt. (online verfügbar unter: http://www.lfu.bayern.de/umweltwissen/doc/uw_19_cu_urlaub_freizeit.pdf).

Stuht, J. N. \& W. G. Youatt (1972): Heartworms and lung flukes from red foxes in Michigan. Journal of Wildlife Management 36(1):166-170. In Sime, C.A. (1999): Domestic Dogs in Wildlife Habitats. In Joslin G., Yousmans H (Hrsg.) (1999): Effects of recreation on Rocky Mountains wildlife: A review for Montana. Committee on Effects of Recreation on Wildlife, Montana Chapter of The Wildlife Society..

Suchant R. (2006): Allianzen für die Zukunft - Modellbeispiele integrativer Lösungen aus dem Schwarzwald. In: Naturverträgliche Steuerung von Tourismus- und FreizeitaktivitätenIntegrierte Lösungen und Konzepte, BayLfU Fachtagung 2006.

Suchant R., Schäfer A. (2002): Integrating Tourism and Grouse Habitat Protection in the Black Forest. In: Monitoring and Management of Visitor Flows in Recreational and Protected Areas, Conference Proceedings, Vienna.

SUM Stadt-Umland-Management Wien/Niederösterreich: http://www.stadt-umland.at/ (aufgerufen am: 24.7.2011).

Szymanska, Ewa (2007): Konflikte zwischen Erholungsnutzung und Naturschutz, Diplomarbeit. Universität für Bodenkultur Wien.

Taczanowska, K., Brandenburg C., Arnberger A., Tomek, H., Muhar, A. (2010): GIS as a tool supporting understanding of visitor flows in recreational ares. In MMV 2010.

Telaar, D. (2007): Geocaching. Eine kontextuelle Untersuchung der deutschsprachigen Geocaching-Community. Diplomarbeit, Westfälische Wilhelms-Universität, Münster.

THIEL D, Jenni-Eiermann S, Jenni L (2008a): Der Einfluss von Freizeitaktivitäten auf das Fluchtverhalten, die Raumnutzung und die Stressphysiologie des Auerhuhns Tetrao urogallus. Der ornithologische Beobachter 105: 85-96.

THIEL D, Jenni-Eiermann S, Jenni L (2008b): Der Einfluss von Freizeitaktivitäten auf das Fluchtverhalten, die Raumnutzung und die Stressphysiologie des Auerhuhns Tetrao urogallus. Der ornithologische Beobachter 105: 85-96.

Thorne, E. T., Kingston N., Jolley W. R., \& Bergstrom R. C. (Hrsg.) (1982): Diseases of wildlife in Wyoming. Second edition. Wyoming Game and Fish Department, Cheyenne, Wyoming. In: Sime, C.A. (1999): Domestic Dogs in Wildlife Habitats. In Joslin G. \& Yousmans H (Hrsg.) (1999): Effects of recreation on Rocky Mountains wildlife: A review for Montana. Committee on Effects of Recreation on Wildlife, Montana Chapter of The Wildlife Society.

Tottewitz, F., Neumann, M., and Sparing, H. (2010): Lebensraumnutzung von Rotwild in der Schorf-heide - Ergebnisse aus mehrjährigen GPS-GSM-Satellitentelemetriestudien. [45], 94- 
106. 2010. Landeskompetenzzentrum Forst Eberswalde (LFE). Eberswalder Forstliche Schriften-reihe. Ref Type: Serial (Book,Monograph).

Umlandverband Frankfurt (UVF) (Hrsg.) (2000a): Landschaftsplan des Umlandverbandes Frankfurt, Band I: Planungs- und Entwicklungskonzeption, Erläuterungen für das Gebiet des Umlandverbandes, Frankfurt.

Umlandverband Frankfurt (UVF) (Hrsg.) (2000b): Landschaftsplan des Umlandverbandes Frankfurt, Band II: Bestandsaufnahmen und sektorale Bewertungen, Erläuterungen für das Gebiet des Umlandverbandes, Frankfurt.

Umlandverband Frankfurt (UVF) (Hrsg.) (2000c): Landschaftsplan UVF. Aufgaben, Ziele, Umsertzung, Frankfurt.

UNEP CBD (1992): Convention on Biological Diversity.

UNEP CBD/COP/7/12 (2004): Decision VII/12 of the Conference of the Parties to the Convention on biological Diversity, 7th meeting, Kuala Lumpur, Malaysia, 9-20 Feb 2004: Sustainable Use (Article 10). In: UNEP/CBD/COP/7/21 2004.

UNESCO \& MAB-ICC (1996): The Seville Strategy and the Statutory Framework of the WorldNetwork of Biosphere Reserves. UNESCO.

Van der Zande, A.N., Berkhuizen, J.C., van Latesteijn, H.C., ter Keurs, W.J.\& Poppelaars, A.J. (1984): Impact of outdoor recreation on the density of a number of breeding bird species in woods adjacent to urban residential areas. Biol. Conserv. 30 In: Piper SD. \& Catterall, CP (2006): Impacts of picnic areas on bird assemblages and nest predation activity within Australian eucalypt forests. In Landscape and Urban Planning 78 (2006) 251-262.

Van der Zee (1987): The recreational resources of the Mae Sa Valley viewed in some theoretical context. (A challenge for further research and reflection). In Proceedings of the seminar on 'The role of geography in the tourism development', pp. 66-68. Geographical Association of Thailand, Kanchanaburi, 26-29 October 2530.

Van der Zee (1990): The complex relationship between landscape and recreation, Landscape Ecology vol. 4, no. 4, pp 225-236.

Verordnung der Luftverkehrsregeln, BGBI. II Nr. 56/1967 i.d.F. Nr. 91/2008.

Verordnung über ein Regionales Raumordnungsprogramm NÖ Mitte vom 21.5.2010. LGBI. 8000/76-2. URL: http://www.ris.bka.gv.at/Dokumente/LrNo/LRNI_2010045/

Verordnung über ein regionales Raumordnungsprogramm nördliches Wiener Umland vom 29.5.2009. LGBI. 8000/86-2. URL: http://www.ris.bka.gv.at/Dokumente/LrNo/LRNI_2009061/

Verordnung über ein Regionales Raumordnungsprogramm südliches Wiener Umland vom 28.4.2010. LGBI. 8000/83-3. URL: http://www.ris.bka.gv.at/Dokumente/LrNo/LRNI_2010037/

Völk, F.; Glitzner, I. \& Wöss, M. (2001): Kostenreduktion bei Grünbrücken durch deren rationellen Einsatz: Kriterien - Indikatoren - Mindeststandards. Straßenforschung, Heft 513. Bundesministerium für Verkehr, Innovation und Technologie, Wien.

WALZ, Ulrich und Alexander BERGER (2004): Analyse der Auswirkungen des Landschaftswandels auf die Erholungseignung. In: Strobl, J, Blaschke, T und Greisebner, G. (Hrsg.): Ange $\neg$ wandte Geoinformatik 2004, Beiträge zum 16. AGIT-Symposium Salzburg 2004, Heidelberg, keine Seitennummern, 11 Seiten, veröffentlicht unter http://www2.ioer.de/recherche/pdf/2004_walz_berger_agit.pdf, 1.11.2009.

Weiss, G. (1991): Naherholungsverhalten von Senioren in der Großstadt

Wiener Jagdgesetz, LGBI. Nr. 34/2001.

Wiener Naturschutzgesetz: LGBI. Nr. 12/2006 idgF.

Wiener Tierschutz- \& Tierhaltegesetz, LGBI. für Wien Nr. 39/ 1987, zuletzt geändert 4/2005. 
Wildauer Lydia, Schreiber Bernd, Reimoser Friedrich (2008): Gutachten zur Anwendung der Richtlinie 79/409/EWG des Rates vom 2. April 1979 über die Erhaltung der wildlebenden Vogelarten.

Wildbiologische Gesellschaft München e.V. (Hrsg.) (1999): Auerhuhnschutz: Aber wie? Ein Leitfaden.

Wolf A. \& Appel-Kummer E. (2009): Naherholung in Stadt und Land, Norderstedt, Books on Demand.

Wrbka et al. (2005): Die Landschaften Österreichs und ihre Bedeutung für die biologische Vielfalt, Umweltbundesamt.

Yalden D.W. \& Yalden P.E. (1990): The sensitivity of breeding golden plovers Pluvialis apricaria to human intruders. Bird Study 36:49-55. In Lenth, B., Brennan, M. \& Knight, L. R. (2006): The Effects of dogs on wildlife communities.

Yalden, P. E. \& D. Yalden (1990): Recreational disturbance of breeding golden plovers Pluvialis apricarius. Biological Conservation 51:243-262. In: Sime, C.A. (1999): Domestic Dogs in Wildlife Habitats. In: Joslin G. \& Yousmans H (Hrsg.) (1999): Effects of recreation on Rocky Mountains wildlife: A review for Montana. Committee on Effects of Recreation on Wildlife, Montana Chapter of The Wildlife Society.

Zentralanstalt für Meteorologie und Geodynamik, www.zamg.ac.at (letzter Zugriff 2010).

ZIENER, Karen (2003): Das Konfliktfeld Erholungsnutzung - Naturschutz in Nationalparken und Biosphärenreservaten (Habilitationsschrift), Aachen, 460 Seiten.

ZIENER, Karen (2005): Auf dem Weg zu einer regionalen und vorausschauenden Konflikt $\urcorner$ strategie für Nationalparke und Biosphärenreservate: Sechs Regionen unter die Lupe genommen (Klagenfurter Geographische Schriften, Heft 25) Klagenfurt, 290 Seiten.

Zweckverband Großraum Braunschweig (Hrsg.) (2008a): Regionales Raumordnungs-programm für den Großraum Braunschweig 2008. Beschreibende Darstellung, Braun-schweig, veröffentlicht unter:

http://www.zgb.de/barrierefrei/misc/downloads/RROP2008_BeschreibendeDarstellung.pdf (aufgerufen am: 2.11.2009).

Zweckverband Großraum Braunschweig (Hrsg.) (2008b): Regionales Raumordnungs-programm für den Großraum Braunschweig 2008 - Begründung, Braunschweig, veröffentlicht unter: http://www.zgb.de/barrierefrei/misc/downloads/RROP2008_Begr\%FCndung.pdf (aufgerufen am: 2.11.2009). 